\title{
Expanding the View: Implications of the SHM Position Statement on Ultrasound Use in Vascular Access
}

\author{
James E Anstey, MD*; Andrew R Lai, MD, MPH
}

Division of Hospital Medicine, Department of Medicine, University of California, San Francisco, San Francisco, California.

$s$ there a single intervention more important to hospitalized patients than vascular access? Since their advent in the 1950s, small plastic tubes have revolutionized medication administration and become a mainstay of modern medicine. Yet, for much of the last 60 years, nurses and doctors have used the same landmark-guided approaches to acquire peripheral and, more specifically, central access. ${ }^{1}$ Minor improvements to the Seldinger technique and sterile preparation have been reported. ${ }^{2}$ However, for such a vital and common procedure, the complication rates of landmark-based approaches to central venous access remain unacceptably high. ${ }^{3}$

In the position statement released by the Society of Hospital Medicine (SHM), Franco-Sadud et al. outline the transformative effects ultrasound can have in obtaining adult vascular access. ${ }^{4}$ The authors cite comprehensive evidence, leaving little doubt of the technique's benefits compared with landmark-based approaches. However, several questions remain: Is vascular access the domain of the hospitalist? If so, how can hospitalists pursue and afford ultrasound training? Finally, how will this shift toward ultrasound-guided vascular access affect patients in resource-limited settings?

Through an expert-driven literature review, the authors present 29 succinct recommendations for ultrasound use in vascular access. Supporting data consistently illustrate the association of ultrasound with increased successful vessel cannulation rates and decreased complication rates for all types of vascular access; including central venous access (internal jugular, subclavian, femoral), arterial line placement, peripherally inserted central catheters, and difficult peripheral venous access. Despite this compelling evidence, however, $20 \%-55 \%$ of all central venous catheters are still placed without ultrasound. ${ }^{5} \mathrm{How}$, then, can hospitalists expand ultrasound use for vascular access or perform these procedures in general?

Hospitalists likely fall into one of three categories in terms of vascular access: (1) they are proficient in ultrasound use for vascular access, (2) they still routinely use traditional landmark-based approaches, or (3) they have little to no involvement in vascular access and defer to intensivists, interventional radiologists, or nurse specialists. Franco-Sadud et al.'s position statement acknowledges the wide range of hospitalist prac-

*Corresponding Author: James E. Anstey, MD; E-mail: james.anstey@ucsf.edu; Telephone: 209-606-1380; Twitter: @jeanstey

Received: July 30, 2019; Revised: August 3, 2019; Accepted: August 6, 2019

() 2019 Society of Hospital Medicine DOI 10.12788/jhm.3299 tices and only asserts that, if providers perform vascular access, they should be trained and use ultrasound to do them. We would advocate further that, regardless of their practice, hospitalists have a role in expanding ultrasound use for vascular access given its direct impact on the patients they care for. Hospitalists who do not directly practice vascular access can still leverage the skills that have established hospital medicine's reputation as leaders in patient safety and quality improvement. Hospitalists can partner with proceduralists in their institutions to ensure that they are supported and trained in the most evidence-based approaches to vascular access and that their patients have access to the highest quality of care.

For the individual hospitalist, the investment of time and resources to incorporate ultrasound into routine practice can seem daunting. In previous position statements, the SHM has advocated for the robust use of simulation and directly observed assessment in credentialing for all bedside procedures. ${ }^{6}$ However, the Society also acknowledges that this degree of training and monitoring can constitute significant barriers and has argued that the onus for change lies not only with providers but with healthcare institutions at large. How, then, can hospitalists approach their institutions to successfully solicit support? While the evidence is not yet conclusive, Cohen et al. have shown promising data for potential long-term cost savings through ultrasound-guided vascular access. ${ }^{7}$ Due to decreased complication rates, downstream benefits of lower resource use, higher patient satisfaction, and, theoretically, even lower clinician burnout rates have been attained. These effects, combined with hospitalists acquiring ultrasound skills translatable to other bedside procedures and fundamentals of diagnostic point of care ultrasound, form a compelling argument for institutional support. Many academic medical centers, typically with increased resources and training programs, have been early adopters; but, how will the shift from landmark-based to ultrasound-guided vascular access affect those in resource-limited settings?

While incredible strides have been made in care quality and patient safety over the last 15 years, improvements clearly do not always benefit patients, clinicians, or institutions equally. ${ }^{8}$ In fact, those in resource-limited settings often experience disproportionately reduced benefits. While focus on the "quality gap" has transformed the culture of the quality improvement and patient safety fields, an "equity gap" has long undermined and limited the impact of those very improvements. Unfortunately, changes in care driven by costly technological advances such as ultrasound are particularly likely to widen this "equity gap." While ultrasound technology is rapidly becoming more 
affordable, a lack of access to machines and appropriate training remain significant barriers in the resource-limited settings that hospitalists are most likely to be performing these procedures. Without a focus on equity, the benefits offered by ultrasound will continue to be limited in their reach.

The SHM position statement by Franco-Sadud et al. is an important step in expanding evidence-based ultrasound use for vascular access and improving patient care. While the recommendations are, at times, aspirational and the barriers are real, hospitalists have shown time and again their ability to overcome these challenges and advance the standard of care for all.

Disclosures: The authors have nothing to disclose.

\section{References}

1. Beheshti MV. A concise history of central venous access. Tech Vasc Interv Radiol. 2011;14(4):184-5. https://doi.org/10.1053/j.tvir.2011.05.002.
2. Higgs ZC, Macafee DA, Braithwaite BD, Maxwell-Armstrong CA. The Seldinger technique: 50 years on. Lancet. 2005;366(9494):1407-1409. https:// doi.org/10.1016/S0140-6736(05)66878-X.

3. Parienti JJ, Mongardon N, Mégarbane B, et al. Intravascular complications of central venous catheterization by insertion site. N Engl J Med. 2015;373(13):1220-1229. https://doi.org/10.1056/NEJMoa1500964.

4. Franco-Sadud R, D Schnobrich, Mathews BK et al. SHM Point-of-care Ultrasound Task Force. Recommendations on the use of ultrasound guidance for central and peripheral vascular access in adults: a position statement of the Society of Hospital Medicine. J Hosp Med. 2019;14:E1-E22. https://doi. org/10.12788/jhm.3287.

5. Soni NJ, Reyes LF, Keyt H, et al. Use of ultrasound guidance for central venous catheterization: a national survey of intensivists and hospitalists. J Crit Care. 2016;36:277-283. https://doi.org/10.1016/j.jcrc.2016.07.014.

6. Lucas BP, Tierney DM, Jensen TP, et al. Credentialing of hospitalists in ultrasound-guided bedside procedures: a position statement of the Society of Hospital Medicine. J Hosp Med. 2018;13(2);117-125. https://doi. org/10.12788/jhm.2917.

7. Cohen ER, Feinglass J, Barsuk JH, et al. Cost savings from reduced catheter-related bloodstream infection after simulation-based education for residents in a medical intensive care unit. Simul Healthc. 2010;5(2):98-102. https://doi.org/10.1097/SIH.0b013e3181bc8304.

8. 2017 National Healthcare Quality and Disparities Report. https://www.ahrq. gov/research/findings/nhqrdr/nhqdr17/index.html. Accessed June 17, 2019. 